\title{
General relativistic solitons. II
}

\author{
A. C. Cadavid \\ Department of Physics and Astronomy, California State University, Northridge, California 91330
}

R. J. Finkelstein

Department of Physics and Astronomy, University of California, Los Angeles, California 90095-1547

(Received 7 July 1998; published 20 May 1999)

\begin{abstract}
We investigate the possible existence of nontopological solitons in stringlike theories, or in other completions of Einstein theory, by examining a simple extension of standard theory that describes a nonlinear scalar field interacting with the Einstein, Maxwell, and Weyl (dilaton) fields. The Einstein and Maxwell couplings are standard while the dilatonic coupling is taken to agree with string models. The nonlinear scalar potential is quite general. It is found to be impossible to satisfy the dilatonic boundary conditions. Excluding the dilaton field we find a variety of solitonic structures differing in ways that depend on the nonlinear potential. In general the excited states exhibit a discrete mass spectrum. At large distances the gravitational field approaches the Reissner-Nordström solution. [S0556-2821(99)04112-0]
\end{abstract}

PACS number(s): 04.62.+v, 04.25.- g, 11.10.Lm

\section{INTRODUCTION}

Since the "black hole solitons" of string and supergravity theories carry horizons and central singularities, they differ from regular solitons which are singularity-free. It is natural to ask if these generally covariant theories also admit regular nontopological solitons, similar to the field structures that exist at the level of special relativity [1,2], since it is known that nontopological solitons also exist at the general relativistic level [3-5]. It is possible, however, that there are generic features of supergravity and of other fundamental completions of the Einstein theory that preclude the existence of everywhere regular solitons that are nontopological. In particular, this role may be played by the dilaton field, which arises as a consequence of Weyl rescaling, couples to the antisymmetric tensor fields, and replaces the numerical coupling constant.

As these theories are derived by compactification from higher dimensional formulations, they characteristically contain multiplets of nonlinear scalar fields that are coupled to multiplets of vector and axial vector fields, and higher tensor fields as well. They are therefore difficult to test for everywhere regular solutions since their field structures, although mitigated by large symmetry groups, are very complex. Nevertheless it may be interesting to examine model theories obtained by coupling the dilaton field and a nonlinear scalar multiplet to the gravitational and electromagnetic fields, both for their intrinsic interest and also for their similarity to more fundamental theories. We shall consider a model theory with the following action which exhibits some of these features:

$$
\begin{aligned}
S= & \int d^{4} x \sqrt{-g}(R-K L), \\
L= & \frac{1}{2} g^{\mu \lambda} \nabla_{\mu} \psi_{0} \nabla_{\lambda} \psi_{0}-\frac{1}{2} e^{\epsilon \psi_{0}} F_{\mu \lambda} F^{\mu \lambda} \\
& +\sum_{i=1}^{N} g^{\mu \lambda} \nabla_{\mu} \psi_{i}\left(\nabla_{\lambda} \psi_{i}\right)^{*}-V\left(\sum_{i=1}^{N} \psi_{i} \psi_{i}^{*}\right),
\end{aligned}
$$

where the neutral scalar $\psi_{0}$ is coupled to $F_{\mu \lambda}$ as a dilaton, where $\epsilon>0$, and where the charged scalars are coupled in the standard way to both the electromagnetic and gravitational fields by the following covariant derivative:

$$
\nabla_{\mu}=\partial_{\mu}+\frac{i e}{c \hbar} A_{\mu}+\Gamma_{\mu}
$$

Here $\Gamma_{\mu}$ is the gravitational part of the covariant derivative and $V$ is the nonlinear scalar interaction. It would be possible to include magnetic couplings in Eq. (1.2) but we shall consider only the case in which magnetic field and magnetic charge vanish. We have not included any self-coupling of the dilaton field. When $\psi_{0}$ is required to be constant, we have a theory with standard couplings.

One may think of this model theory as interpolating between standard physics $\psi_{0}=$ const, and new physics associated with a Kaluza-Klein theory. It is not, however, intended as a truncation of a supergravity theory.

\section{THE SOLITON PROBLEM}

In the action (1.1) there are the following coupled fields: the metric $g_{\mu \lambda}$, the vector potential $A_{\mu}$, and the scalar fields $\psi_{i}$. The equations of motion are

$$
\begin{aligned}
\frac{\delta S}{\delta g_{\mu \lambda}} & =0, \\
\frac{\delta S}{\delta A_{\mu}} & =0, \\
\frac{\delta S}{\delta \psi_{i}} & =0, \quad i=0, \ldots, N .
\end{aligned}
$$

We require everywhere regular solutions of these equations subject to solitonic boundary conditions. Therefore no central singularities or horizons are permitted. These solitonic solutions concentrate energy momentum and charge 
current within lumps of field. In general, the mass spectrum of these particlelike lumps may be discrete.

In this theory both the nonlinear scalar fields and the Maxwell field contribute to the source of the gravitational field while the complex scalars are the source of the electromagnetic field. To avoid irrelevant complications, it is assumed that the charged scalar fields are spherically symmetric and harmonically time dependent, while the dilaton is also spherically symmetric but time independent:

$$
\begin{aligned}
& \psi_{0}(x)=R_{0}(r), \\
& \psi_{i}(x)=e^{i \omega t} R_{i}(r), \quad i=1, \ldots N .
\end{aligned}
$$

We shall need to consider only one member of the $S U(N)$ scalar multiplet.

Since we are dropping the magnetic fields, the vector potential also vanishes and the scalar potential is spherically symmetric. Thus the electric and gravitational fields should resemble the Reissner-Nordström solution except near the origin where the mass and charge are spread out and all fields remain finite.

\section{THE FIELD EQUATIONS}

The field equations implied by Eqs. (1.2) and (1.3) may be expressed as follows.

(a) The gravitational equations

$$
R_{\mu \lambda}=K \Theta_{\mu \lambda} \quad K=-\frac{8 \pi k}{c^{2}},
$$

where $k$ is Newton's constant. Here

$$
\begin{gathered}
\Theta_{\mu \lambda}=\theta_{\mu \lambda}-\frac{1}{2} \theta g_{\mu \lambda}, \\
\theta_{\mu \lambda}=\frac{\partial L}{\partial g^{\mu \lambda}}-\frac{1}{2} g_{\mu \lambda} L .
\end{gathered}
$$

(b) The scalar equations

$$
\begin{array}{r}
g^{\mu \lambda} \nabla_{\mu} \nabla_{\lambda} \psi_{0}+\frac{\epsilon}{2} e^{\epsilon \psi_{0}} F_{\mu \lambda} F^{\mu \lambda}=0, \\
g^{\mu \lambda} \nabla_{\mu} \nabla_{\lambda} \psi_{i}+\frac{\partial V}{\partial \psi_{i}^{*}}=0 .
\end{array}
$$

The operator $\nabla_{\mu}$ contains the $A_{\mu}$ term in Eq. (3.5) but not in Eq. (3.4).

(c) The electromagnetic equations

$$
\begin{aligned}
& \frac{1}{\sqrt{-g}} \partial_{\lambda}\left(\sqrt{-g} e^{\epsilon \psi_{0}} F^{\mu \lambda}\right) \\
& \quad=\frac{i e}{4 \hbar c} \sum\left[\psi_{i}\left(\nabla^{\mu} \psi_{i}\right)^{*}-\psi_{i}^{*}\left(\nabla^{\mu} \psi_{i}\right)\right] .
\end{aligned}
$$

The usual equation for the conserved current follows from Eq. (3.6): namely,

$$
\partial_{\mu}\left\{\sqrt{-g} \sum_{i=1}^{N}\left[\psi_{i}\left(\nabla^{\mu} \psi_{i}\right)^{*}-\psi_{i}^{*}\left(\nabla^{\mu} \psi_{i}\right)\right]\right\}=0 .
$$

To simplify the general equations (3.1), choose the line element

$$
d s^{2}=e^{\nu(r)} c^{2} d t^{2}-e^{\lambda(r)} d r^{2}-r^{2}\left(d \theta^{2}+\sin ^{2} \theta d \varphi^{2}\right)
$$

and in Eqs. (3.4) and (3.5) choose

$$
F_{\alpha \beta}=\left(\begin{array}{cccc}
0 & -E & 0 & 0 \\
E & 0 & 0 & 0 \\
0 & 0 & 0 & 0 \\
0 & 0 & 0 & 0
\end{array}\right),
$$

where

$$
E=-\frac{\partial \varphi}{\partial r}=-\varphi^{\prime}
$$

and $\varphi$ is $A_{0}$, the scalar potential of the electromagnetic field. The complete set of gravitational equations is now

$$
\begin{aligned}
-\frac{\nu^{\prime \prime}}{2} & +\frac{\nu^{\prime} \lambda^{\prime}}{4}-\frac{\left(\nu^{\prime}\right)^{2}}{4}-\frac{\nu^{\prime}}{r} \\
& =K\left[e^{\lambda-\nu}\left(\frac{\omega}{c}+\frac{e}{\hbar c} \varphi\right)^{2} R^{2}-\frac{e^{\lambda}}{2} V\left(R^{2}\right)+\Delta\right],
\end{aligned}
$$

$$
\begin{aligned}
\frac{\nu^{\prime \prime}}{2}- & \frac{\nu^{\prime} \lambda^{\prime}}{4}+\frac{\left(\nu^{\prime}\right)^{2}}{4}-\frac{\lambda^{\prime}}{r} \\
& =K\left[\frac{1}{2}\left(R_{0}^{\prime}\right)^{2}+\left(R^{\prime}\right)^{2}+\frac{1}{2} e^{\lambda} V\left(R^{2}\right)-\Delta\right], \\
\left(\nu^{\prime}-\lambda^{\prime}\right) \frac{r}{2} & =K\left[\frac{r^{2}}{2} V\left(R^{2}\right) e^{\lambda}+r^{2} \Delta\right]+e^{\lambda}-1 .
\end{aligned}
$$

The off-diagonal equations are satisfied identically.

Here

$$
\begin{aligned}
\Delta & =e^{\epsilon R_{0}-\nu}\left(\varphi^{\prime}\right)^{2} / 2 c^{2}, \\
R^{2} & =\sum_{1}^{N} R_{i}^{2} .
\end{aligned}
$$

The equations for the dilaton and the charged scalars are

$$
\begin{gathered}
R_{0}^{\prime \prime}+\frac{2}{r} R_{0}^{\prime}+\frac{\nu^{\prime}-\lambda^{\prime}}{2} R_{0}^{\prime}+\epsilon e^{\epsilon R_{0}-\nu}\left(\varphi^{\prime}\right)^{2}=0, \\
R_{i}^{\prime \prime}+\frac{2}{r} R_{i}^{\prime}+\frac{\nu^{\prime}-\lambda^{\prime}}{2} R_{i}^{\prime}+\left(\frac{\omega}{c}+\frac{e}{\hbar c} \varphi\right)^{2} e^{\lambda-\nu} R_{i} \\
-e^{\lambda} \frac{\partial}{\partial R_{i}} V\left(R^{2}\right)=0, \quad i=1 \ldots N .
\end{gathered}
$$


Finally the single equation for the electromagnetic scalar potential is

$$
\begin{aligned}
\varphi^{\prime \prime} & +\frac{2}{r} \varphi^{\prime}-\left[\frac{1}{2}\left(\nu^{\prime}+\lambda^{\prime}\right)+\epsilon R_{0}^{\prime}\right] \varphi^{\prime} \\
& =-\frac{1}{2} \frac{e}{\hbar c}\left(\frac{\omega}{c}+\frac{e}{\hbar c} \varphi\right) e^{-\epsilon R_{0}+\lambda} R^{2} .
\end{aligned}
$$

The boundary conditions may be chosen as follows:

$$
\begin{aligned}
& R_{i}^{\prime}(0)=\varphi^{\prime}(0)=0, \\
& R_{i}(\infty)=\lambda(\infty)=\nu(\infty)=\varphi(\infty)=0, \\
& R_{i}^{\prime}(\infty)=\lambda^{\prime}(\infty)=\nu^{\prime}(\infty)=\varphi^{\prime}(\infty)=0,
\end{aligned}
$$

where $i=0, \ldots, N$. In addition we require

$$
V(\infty)=0 .
$$

Since the $R_{i}$ are scalars, the equations (3.19) are invariant conditions which exclude cusps at the origin in these fields.

The corresponding restriction on $\varphi$ is

$$
\varphi^{\prime}(0)=E(0)=0,
$$

which is required since the origin is at the center of a spherically symmetric charge distribution. In invariant form Eq. (3.23a) reads

$$
\left(F_{\mu \lambda} F^{\mu \lambda}\right)_{r=0}=0 .
$$

In the rest of this paper we shall use the gauge where

$$
\begin{aligned}
& R_{i}=0, \quad i=2, \ldots, N, \\
& R_{1}=R .
\end{aligned}
$$

We shall also need the following relation;

$$
\begin{aligned}
-\frac{\left(\nu^{\prime}+\lambda^{\prime}\right)}{r}= & K\left[\left(R^{\prime}\right)^{2}+\frac{1}{2}\left(R_{0}^{\prime}\right)^{2}\right. \\
& \left.+\left(\frac{\omega}{c}+\frac{e}{\hbar c} \varphi\right)^{2} e^{\lambda-\nu} R^{2} / c^{2}\right]
\end{aligned}
$$

obtained by adding Eqs. (3.11) and (3.12). It follows from the preceding equation and the boundary conditions that

$$
\nu^{\prime}(0)+\lambda^{\prime}(0)=0 .
$$

The invariant form of Eq. (3.26) may be expressed in terms of $g$, the determinant of the metric tensor, as follows:

$$
\left(\frac{g}{g_{\text {flat }}}\right)_{r=0}^{\prime}=0 .
$$

One also notes that Eq. (3.13) implies

$$
\nu^{\prime}(0)-\lambda^{\prime}(0)=\left(\frac{2}{r}\left(e^{\lambda}-1\right)\right)_{r=0} .
$$

Here

$$
\lambda=l_{0}+l_{2} r^{2}+\cdots
$$

since $\lambda(r)$ and $\nu(r)$ are even by Eqs. (3.11)-(3.13). If

$$
e^{\lambda(0)} \neq 1
$$

then by Eq. (3.28)

$$
\nu^{\prime}(0)-\lambda^{\prime}(0)=\infty
$$

and by Eq. (3.26) both $\nu(r)$ and $\lambda(r)$ would have infinite cusps at $r=0$. If $\lambda(0)$ vanishes, however, then, by Eq. (3.28),

$$
\nu^{\prime}(0)-\lambda^{\prime}(0)=0 .
$$

Therefore we adopt the following boundary conditions:

$$
\begin{gathered}
\lambda(0)=0, \\
\lambda^{\prime}(0)=\nu^{\prime}(0)=0 .
\end{gathered}
$$

These boundary conditions are compatible with Eqs. (3.26) and (3.32) as well as with Eqs. (3.11), (3.12), and (3.13). Finally by Eq. (3.25) we have

$$
\begin{aligned}
{[\nu+\lambda]_{0}^{\infty}=} & -K \int_{0}^{\infty} d r r\left[\left(R^{\prime}\right)^{2}+\frac{1}{2}\left(R_{0}^{\prime}\right)^{2}\right. \\
& \left.+\left(\frac{\omega}{c}+\frac{e}{\hbar c} \varphi\right)^{2} e^{\lambda-\nu} \frac{R^{2}}{c^{2}}\right]
\end{aligned}
$$

and since the integrand is positive, while $K$ is negative,

$$
[\nu+\lambda]_{0}^{\infty}>0 .
$$

Then by Eq. (3.20)

$$
\nu(0)+\lambda(0)<0 .
$$

By Eq. (3.33)

$$
\nu(0)<0 .
$$

\section{APPROXIMATE SOLUTIONS}

The boundary conditions (3.19) and (3.20) imply

$$
\lim _{\substack{r \rightarrow 0 \\ r \rightarrow \infty}} \Delta(r)=0 .
$$

Therefore Eqs. (3.11)-(3.13) all approach the corresponding equations (except for the frequency shift $\omega+e \varphi / \hbar$ ) that hold in the absence of a charged source. These equations were previously studied [4]. Their solutions remain finite and have flat tangents at $r=0$.

\section{A. The electromagnetic scalar potential}

Let us consider Eq. (3.18) near $r=0$. Guided by earlier results [4], let us set 


$$
\begin{aligned}
R_{0} & =\hat{a}_{0}+\hat{a}_{2} r^{2}+\cdots, \\
R & =a_{0}+a_{2} r^{2}+\cdots, \\
\lambda & =l_{2} r^{2}+l_{4} r^{4}+\cdots, \\
\nu & =n_{0}+n_{2} r^{2}+n_{4} r^{4}+\cdots .
\end{aligned}
$$

Then

$$
R_{0}^{\prime}(0)=R^{\prime}(0)=\lambda^{\prime}(0)=\nu^{\prime}(0)=0
$$

and Eq. (3.18) near $r=0$ becomes

$$
\varphi^{\prime \prime}+\frac{2}{r} \varphi^{\prime} \cong-\frac{e}{2 \hbar c}\left(\frac{\omega}{c}+\frac{e \varphi}{\hbar c}\right) e^{-\epsilon \hat{a}_{0}} a_{0}^{2} .
$$

Set

$$
y=\frac{\omega}{c}+\frac{e \varphi}{\hbar c}
$$

Now Eq. (4.7) becomes

$$
y^{\prime \prime}+\frac{2}{r} y^{\prime} \cong-\frac{1}{2} \frac{e^{2}}{\hbar^{2} c^{2}} e^{-\epsilon \hat{a}_{0}} a_{0}^{2} y .
$$

The solution of Eq. (4.9) is

$$
y=\frac{\sin p r}{r},
$$

where

$$
p^{2}=\frac{e^{2}}{2 \hbar^{2} c^{2}} e^{-\epsilon \hat{a}_{0}} a_{0}^{2} .
$$

Therefore

$$
\lim _{r \rightarrow 0} \varphi=\frac{1}{\sqrt{2}} \exp \left[-\epsilon \frac{\hat{a}_{0}}{2}\right] a_{0}-\frac{\hbar}{e} \omega .
$$

Hence all fields including $\varphi(r)$ remain finite with vanishing derivatives at $r=0$. On the other hand at very large distances Eq. (3.18) becomes

$$
\varphi^{\prime \prime}+\frac{2}{r} \varphi^{\prime}=0
$$

and

$$
\varphi=\frac{Q}{r}
$$

where $Q$ is the charge.

\section{B. The dilaton}

Let us next consider the equation of the dilaton (3.16). Since all first derivatives vanish at the origin, it follows from this equation that $R_{0}^{\prime \prime}$ and $R_{0}^{\prime \prime \prime}$ also vanish at this point. The first nonvanishing derivative at the origin is

$$
R_{0}^{I V}(0)=-2 \epsilon e^{-\nu(0)+\epsilon R_{0}(0)}\left[\varphi^{\prime \prime}(0)\right]^{2} .
$$

Let us first assume that $\epsilon>0$. Then $R_{0}(r)$ has a very flat maximum at the origin, and therefore near the origin

$$
R_{0}^{\prime}(r)<0 \text {. }
$$

Equation (3.16) also implies that $R_{0}(r)$ has no minimum anywhere since

$$
R_{0}^{\prime \prime}(r)=-\epsilon e^{-\nu+\epsilon R_{0}}\left(\phi^{\prime}\right)^{2} \leqslant 0
$$

wherever $R_{0}^{\prime}(r)$ vanishes.

To determine the behavior of $R_{0}(r)$ at very large $r$ one may anticipate the result that $\lambda$ and $\nu$ both decrease as $1 / r$. Then Eq. (3.16) becomes approximately

$$
R_{0}^{\prime \prime}(r)+\frac{2}{r} R_{0}^{\prime}(r)+\epsilon\left(\phi^{\prime}\right)^{2} \cong 0
$$

and if one sets

$$
R_{0}=A r^{s}+B
$$

$$
\phi=\frac{Q}{r}
$$

one finds by Eq. (4.17)

$$
R_{0}(r)=\frac{-\epsilon Q^{2}}{2 r^{2}}+B
$$

To satisfy the boundary condition at infinity set $B=0$. By Eq. (4.20a) one has at large $r$

$$
R_{0}^{\prime}(r)=\frac{\epsilon Q^{2}}{r^{3}}>0
$$

Now Eqs. (4.15b) and (4.20b) are in conflict unless there is an intervening minimum. But Eq. (4.16) does not allow a minimum anywhere, and so it is not possible to satisfy the boundary conditions at both $r=0$ and $r=\infty$. If we assume the opposite sign of $\epsilon$, then there will be a minimum instead of a maximum at the origin and all the inequalities will be reversed; but there will remain the same incompatibility between the boundary conditions at the origin and at infinity. Since the presence of the dilaton field appearing in Eq. (1.2) therefore precludes the existence of a nontopological soliton, we shall set $\psi_{0}=0$ in Eq. (1.2) and delete $R_{0}$ from all the equations following this paragraph. The resulting action and equations are then standard, but the potential remains general.

\section{The gravitational equations}

Let us next examine the set (3.11)-(3.13) at $r=\infty$ subject to the boundary conditions (3.20) and (3.21). (We shall an- 
ticipate the result that $R$ vanishes faster than $\nu$ and $\lambda$ as $r$ $\rightarrow \infty$.) Then by Eq. (3.25) we have, at large $r$,

$$
\nu^{\prime}+\lambda^{\prime} \cong 0
$$

By Eq. (3.20)

$$
\nu(\infty)+\lambda(\infty)=0 .
$$

Therefore at large distances

$$
\nu+\lambda \cong 0 .
$$

Then by Eq. (3.13)

$$
-\frac{\lambda^{\prime}}{r} \rightarrow K\left[\frac{1}{2} V\left(R^{2}\right) e^{\lambda}+\frac{e^{\lambda}}{2 c^{2}}\left(\varphi^{\prime}\right)^{2}\right]+\frac{e^{\lambda}-1}{r^{2}},
$$

where we have also substituted Eqs. (3.14) and (4.23).

By the boundary conditions $V\left(R^{2}\right)$ vanishes at infinity. We know that $\lambda$ vanishes as $1 / r$ and it will be seen that $R$ vanishes as a spherical Bessel function. Then Eq. (4.24) becomes

$$
-\frac{\lambda^{\prime}}{r} \cong \frac{K}{2 c^{2}}\left(\varphi^{\prime}\right)^{2} e^{\lambda}+\frac{e^{\lambda}-1}{r^{2}} .
$$

To second order in $1 / r$, let us set

$$
\begin{aligned}
& \lambda \cong \frac{2 m}{r}+\frac{l_{2}}{r^{2}}, \\
& \varphi \cong \frac{Q}{r}+\frac{q_{2}}{r^{2}} .
\end{aligned}
$$

Then by examining terms of order $1 / r^{4}$ one sees that

$$
l_{2}=2 m^{2}+\frac{K Q^{2}}{2 c^{2}} \text {. }
$$

Note that

$$
e^{\nu}=\left(e^{-\lambda}\right)=1-\frac{2 m}{r}+\frac{2 m^{2}-l_{2}}{r^{2}}
$$

Then by Eq. (4.28)

$$
e^{\nu}=1-\frac{2 m}{r}-\frac{K Q^{2}}{2 c^{2} r^{2}}
$$

where $m$ and $Q$ are mass and charge. The terms in $1 / r$ and $1 / r^{2}$ agree with the Reissner-Nordström result for a point source. For the present case of an extended source, there will be additional terms of higher order in $1 / r$.

\section{Charged scalars}

In order to extract similar asymptotic relations from Eq. (3.17), it is sufficient to assume

$$
V\left(R^{2}\right)=\frac{B}{2} R^{2}+\cdots .
$$

Then at large $r$ one has

$$
R^{\prime \prime}+\frac{2}{r} R^{\prime}+\frac{\nu^{\prime}-\lambda^{\prime}}{2} R^{\prime}+\left(\frac{\omega}{c}+\frac{e \varphi}{\hbar c}\right)^{2} e^{\lambda-\nu} R-e^{\lambda} B R=0 .
$$

Let us carry $R$ to the same order as $\lambda$ in $1 / r$. Therefore, let us make the ansatz

$$
R=\frac{e^{-\alpha r}}{r}\left(1+\frac{p_{1}}{r}\right)
$$

Then

$$
R^{\prime \prime}+\frac{2}{r} R^{\prime}=\left(\alpha^{2}+\frac{2 p_{1} \alpha}{r^{2}}\right) R
$$

Equation (4.32) may now be written to order $\left(1 / r^{2}\right) R$ as follows:

$$
\begin{gathered}
\left(\alpha^{2}+\frac{2 p_{1} \alpha}{r^{2}}\right)-\frac{2 m \alpha}{r^{2}}+\left(1+\frac{4 m}{r}+\frac{2 l_{2}+8 m^{2}}{r^{2}}\right) \frac{\omega^{2}}{c^{2}} \\
+\frac{2 e}{\hbar c^{2}} Q \omega\left(\frac{1}{r}+\frac{4 m}{r^{2}}\right)+\frac{2 e \omega q_{2}}{\hbar c^{2} r^{2}}+\frac{e^{2} Q^{2}}{\hbar^{2} c^{2} r^{2}} \\
-\left(1+\frac{2 m}{r}+\frac{l_{2}+2 m^{2}}{r^{2}}\right) B=0
\end{gathered}
$$

From Eq. (4.35) one finds

$$
\begin{gathered}
B=\alpha^{2}+\frac{\omega^{2}}{c^{2}} \geqslant 0, \\
B=\frac{2 \omega^{2}}{c^{2}}+\frac{e}{\hbar c^{2}} \frac{Q \omega}{m}, \\
\left(p_{1}-m\right) \alpha+2 m^{2} \alpha^{2}+g(Q)=0,
\end{gathered}
$$

where

$$
g(Q)=\frac{e^{2}}{2 \hbar^{2} c^{2}} Q^{2}-\frac{K \omega}{2 m} \frac{e}{\hbar c^{2}} Q^{3}+\frac{e \omega}{c} q_{2} .
$$

By Eqs. (4.36) and (4.37) the tail of $R(r)$ is determined by

$$
\alpha^{2}=\frac{\omega^{2}}{c^{2}}+\frac{l \omega}{\hbar c^{2} m} Q .
$$

If the mass is very large

$$
\alpha \cong \frac{\omega}{c} .
$$

\section{THE SPECIAL RELATIVISTIC EIGENVALUE PROBLEM}

The general relativistic equations that must be satisfied are Eqs. (3.11)-(3.18) subject to the boundary conditions (3.19)-(3.22) as well as Eqs. (3.33) and (3.34), after the dilaton equations have been deleted. The qualitative nature 
of the solutions to these equations is already determined by the eigenvalue problem generated by the special relativistic neutral limit of Eq. (3.17), namely,

$$
R^{\prime \prime}+\frac{2}{r} R^{\prime}+\frac{\omega^{2}}{c^{2}} R-\frac{\partial V}{\partial R}=0
$$

which holds in the limit at large distances.

To discuss Eq. (5.1) introduce the auxiliary function

$$
H=\frac{1}{2}\left(R^{\prime}\right)^{2}-V\left(R^{2}\right)+\frac{1}{2} \frac{\omega^{2}}{c^{2}} R^{2} .
$$

By Eq. (5.1),

$$
\frac{d H}{d r}=-\frac{2}{r}\left(R^{\prime}\right)^{2} \leqslant 0 .
$$

It follows that the representative point in the phase plane (the $R R^{\prime}$ plane) will always move towards lower values of $H$ unless $R^{\prime}=0$. Hence, if $H\left(R, R^{\prime}\right)$ is pictured as a surface above the $R R^{\prime}$ plane, the representative point will fall toward minima on this surface.

The extrema in the $R^{\prime}$ direction are given by

$$
\frac{\partial H}{\partial R^{\prime}}=R^{\prime}=0
$$

This locus is the $R$ axis. It is a minimum in the $R^{\prime}$ direction since

$$
\frac{\partial^{2} H}{\partial\left(R^{\prime}\right)^{2}}=1>0 .
$$

The extrema in the $R$ direction depend on $V\left(R^{2}\right)$.

For definiteness assume that $V\left(R^{2}\right)$ may be represented by the polynomial

$$
V\left(R^{2}\right)=\frac{B}{2} R^{2}+\frac{C}{4} R^{4}+\frac{D}{6} R^{6}
$$

where

$$
B>0, \quad C<0, \quad D>0 .
$$

Since $D$ is positive, the field energy is bounded below. Then

$$
\begin{gathered}
\frac{\partial H}{\partial R}=-\frac{\partial V}{\partial R}+\frac{\omega^{2}}{c^{2}} R=-\left(B R+C R^{3}+D R^{5}\right)+\frac{\omega^{2}}{c^{2}} R, \\
\frac{\partial^{2} H}{\partial R^{2}}=-\frac{\partial^{2} V}{\partial R^{2}}+\frac{\omega^{2}}{c^{2}}=-\left(B+3 C R^{2}+5 D R^{4}\right)+\frac{\omega^{2}}{c^{2}} .
\end{gathered}
$$

By Eq. (5.8) the extrema in the $R$ direction lie at the roots of

$$
\frac{\partial H}{\partial R}=\left(\widetilde{B}+C R^{2}+D R^{4}\right) R=0
$$

where

$$
\widetilde{B}=B-\frac{\omega^{2}}{c^{2}} .
$$

The five roots of Eq. (5.10) are

$$
R=0
$$

and

$$
\begin{array}{ll}
R_{++}=+\left\{\frac{|C|+E}{2 D}\right\}^{1 / 2}, & R_{-+}=-R_{++}, \\
R_{+-}=+\left\{\frac{|C|-E}{2 D}\right\}^{1 / 2}, & R_{--}=-R_{+-} .
\end{array}
$$

Here

$$
E^{2}=C^{2}-4 D \widetilde{B}
$$

Then

$$
-\left(\frac{\partial^{2} H}{\partial R^{2}}\right)_{R=0}=\widetilde{B} .
$$

We now require $\widetilde{B}=B-\omega^{2} / c^{2}>0$. Then the origin is a maximum in the $R$ direction.

At $R_{++}$and $R_{+-}$we have

$$
\begin{aligned}
& \left(\frac{\partial^{2} H}{\partial R^{2}}\right)_{++}=-\frac{E}{D}(|C|+E)<0, \\
& \left(\frac{\partial^{2} H}{\partial R^{2}}\right)_{+-}=\frac{E}{D}(|C|-E)>0
\end{aligned}
$$

since $|C|>E$. Hence the points $\left(R_{+-}, 0\right)$ and $\left(-R_{+-}, 0\right)$ are minima in both $R$ and $R^{\prime}$ directions. The point $(0,0)$ is a minimum in the $R^{\prime}$ direction but a maximum in the $R$ direction and is therefore a saddle point. Similarly the points $\left(R_{++}, 0\right)$ and $\left(-R_{++}, 0\right)$ are saddle points.

The phase portrait of $H\left(R, R^{\prime}\right)$ is shown in Fig. 1. We shall assume in this figure that

$$
H\left(R_{++}, 0\right)>H(0,0) \text { or } H\left(R_{++}, 0\right)>0 .
$$

To satisfy this condition, $D$ is restricted by the following inequality:

$$
\frac{3}{16} \frac{C^{2}}{\widetilde{B}} \geqslant D
$$

If $V(R)$ were a higher order polynomial or some other functional, the phase portrait could have more structure than is shown in the figure. It will become clear, however, that only that portion of the surface near the origin is relevant here.

Let $C_{0}$ and $C_{++}$be iso- $H$ curves passing through $(0,0)$ and $\left(R_{++}, 0\right)$. Require that $D$ be restricted by Eq. (5.20). Then if the initial point of the solution curve lies anywhere in 


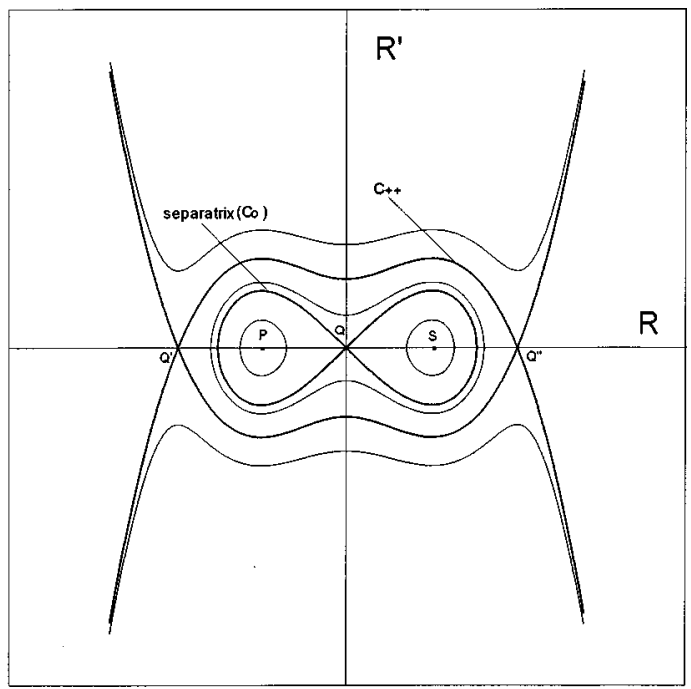

FIG. 1. Phase portrait of $H\left(R, R^{\prime}\right) . Q, Q^{\prime}$, and $Q^{\prime \prime}$ are saddle points. $S$ and $P$ are minima. The $R$ coordinates of $Q^{\prime}, Q$, and $Q^{\prime \prime}$ are $R_{-+}, 0$, and $R_{++}$, respectively. The $R$ coordinates of $S$ and $P$ are $R_{+-}$and $R_{--}$, respectively.

the phase plane between $C_{0}$ and $C_{++}$, this curve must terminate, according to Eq. $(5.3)$, at $(0,0),\left(R_{+-}, 0\right)$, or $\left(-R_{+-}, 0\right)$. In the soliton problem the initial point of the solution curve must lie on the $R$ axis, and if it also lies between $C_{0}$ and $R_{++}$it must terminate at either one of the two attractors, $R_{+-}$and $-R_{+-}$, or at the origin.

In Fig. 2 the region between $C_{0}$ and $C_{++}$is expanded. The solution curves are shown starting at $p_{0}, s_{0}$, and $q_{0}$ and ending at $P, S$, and $Q$. In Fig. 3 the same solutions are shown in configuration space.

Every solution curve starting on the axis between $R_{+-}$ and $R_{++}$and, depending on its initial point, lies in one of two classes going to either the left $(P)$ or right $(S)$ attractor, unless it goes to the origin. The relation between curves in the $p$ and $s$ classes and the eigensolution is

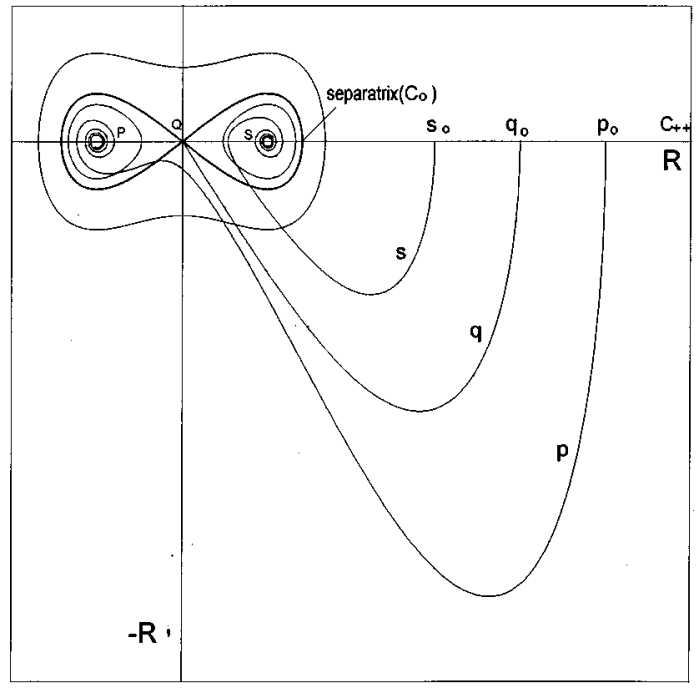

FIG. 2. Solution curves $s$ and $p$ bounding the eigensolution $q$, shown in the phase plane.

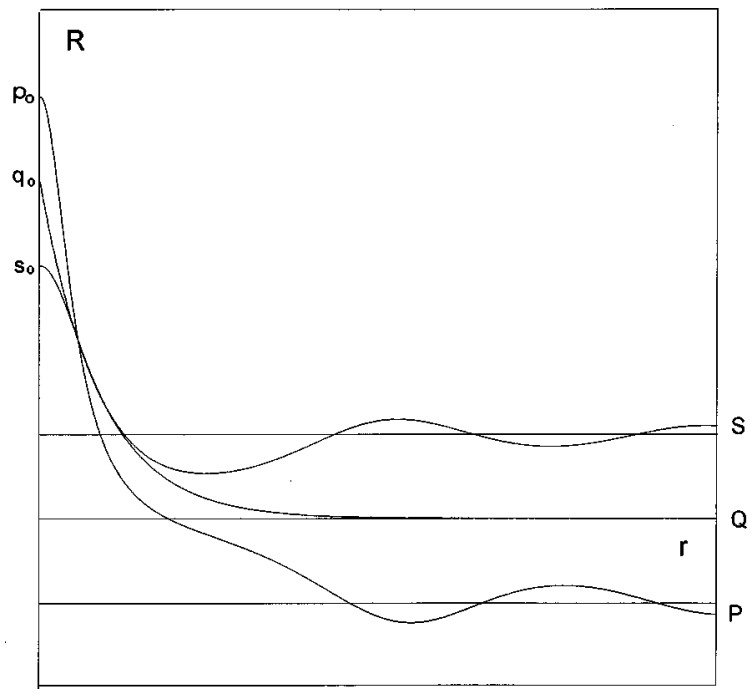

FIG. 3. Solution curves $s$ and $p$ bounding the eigensolution $q$, shown in configuration space.

$$
\lim _{p_{0}-s_{0} \rightarrow 0}\left[\frac{R(p)+R(s)}{2}\right]=R(q),
$$

where $p, s$, and $q$ refer to the three curves going to $P, S$, and $Q$. By bringing $p_{0}$ and $s_{0}$ together one may determine the eigenvalue $q_{0}$ with arbitrary accuracy. Similarly Eq. (5.21) is a prescription for obtaining the eigensolutions $R(q)$ with arbitrary accuracy.

Note that at large $r$ we have approximately

$$
\begin{aligned}
& R(p)=A+u(p), \\
& R(s)=-A+u(s), \\
& R(q)=u(q),
\end{aligned}
$$

where $A=R_{+-}$. Since $( \pm A, 0)$ are constant solutions of Eq. (5.1), they are also roots of the following polynomial and hence extrema of $H$ :

$$
\left(\frac{\partial H}{\partial R}\right)_{\bar{R}}=\frac{\omega^{2}}{c^{2}} \bar{R}-\left(\frac{\partial V}{\partial R}\right)_{\bar{R}}=0, \quad \bar{R}=( \pm A, 0) .
$$

Here $u_{p}, u_{s}$, and $u_{q}$ are spherical Bessel functions that solve Eq. (5.1) after it is linearized near $\pm A$ and zero. The linearized equation is

$$
u^{\prime \prime}+\frac{2}{r} u^{\prime}+\left(\frac{\partial^{2} H}{\partial R^{2}}\right)_{\bar{R}} u=0, \quad \bar{R}=( \pm A, 0)
$$

and

$$
\begin{gathered}
u(p)=\frac{a_{p}}{r} \sin \left(\widetilde{\omega}_{p} r+\epsilon_{p}\right), \\
u(s)=\frac{a_{s}}{r} \sin \left(\widetilde{\omega}_{s} r+\epsilon_{s}\right),
\end{gathered}
$$




$$
u(q)=\frac{a_{q}}{r} e^{-\alpha r}
$$

where

$$
\begin{aligned}
\widetilde{\omega}_{p}^{2} & =\widetilde{\omega}_{s}^{2}=\left(\frac{\partial^{2} H}{\partial R^{2}}\right)_{A}, \\
\alpha^{2} & =-\left(\frac{\partial^{2} H}{\partial R^{2}}\right)_{0} .
\end{aligned}
$$

The functions $u(p)$ and $u(s)$ describe the spiral motion toward the attractors while $u(q)$ describes the exponential approach to the saddle point. Note that the oscillations of $u(p)$ and $u(s)$ begin to cancel in the sum $\frac{1}{2}[R(p)+R(s)]$ because of the phase difference between the arguments of $u(p)$ and $u(s)$ that arise because the motions around $S$ and $P$ are clockwise and counterclockwise, respectively, $\left(\widetilde{\omega}_{p}\right.$ $\left.=-\widetilde{\omega}_{s}\right)$. In the large distance limit the oscillatory behavior disappears since $\frac{1}{2}[u(p)+u(s)]$ must approach $u(q)$.

As $p_{0}$ and $s_{0}$ are brought closer to $q_{0}$ the oscillatory behavior of $p$ and $s$ recedes to infinity and both $p$ and $s$ approach the eigensolution $q$ which terminates at the origin with no oscillations. The preceding discussion continues to hold as the initial values, $p$ and $s$, are increased. It may then happen that the $p$ and $s$ curves cross the $R^{\prime}$ axis several times before being caught on one of the attractors. In that case the eigensolutions will node a corresponding number of times and the resultant field structure may be described as an excited state of the soliton.

On the other hand, if the initial point lies inside the separatrix, the solution curve will always end at the nearby attractor. According to one of the boundary conditions that we have imposed, namely, $R(\infty)=0$, such a curve is not an eigensolution. If the boundary conditions were relaxed, however, so that $R(\infty)$ is interpreted as a vacuum expectation value, as in the case with gauge solitons, this interior curve could be regarded as belonging to a continuum of eigenstates.

The preceding qualitative discussion is based entirely on Eq. (5.1). In the next section we will present the numerical solution to the coupled equations (3.13), (3.17), (3.18), and (3.25) with the dilaton contribution set to zero.

\section{THE GENERAL RELATIVISTIC EIGENVALUE PROBLEM}

Although we have not found an $H$ functional appropriate to the full set of equations, nevertheless the numerically obtained solutions of the original equation (3.17) behave qualitatively in the way just described for the simplified equation (5.1). We may gain additional information by regarding the full equation (3.17) as a perturbation on Eq. (5.1) and by confining the discussion to the $R R^{\prime}$ plane.

Consider, for example, the region of phase space where $\nu=\lambda=\nu^{\prime}=\lambda^{\prime} \simeq 0$. Then

$$
R^{\prime \prime}+\frac{2}{r} R^{\prime}+\left(\frac{\omega}{c}+\frac{e}{\hbar c} \varphi\right)^{2} R-\frac{\partial V}{\partial R}=0
$$

and

$$
\begin{aligned}
\frac{d H}{d r} & =R^{\prime}\left[R^{\prime \prime}+\omega^{2} R-\frac{\partial V}{\partial R}\right] \\
& =\left(R^{\prime}\right)^{2}\left\{-\frac{2}{r}-\left[\left(\frac{\omega}{c}+\frac{e}{\hbar c} \varphi\right)^{2}-\frac{\omega^{2}}{c^{2}}\right] \frac{R}{R^{\prime}}\right\} .
\end{aligned}
$$

For very large $R$

$$
\frac{d H}{d r} \cong \frac{2\left(R^{\prime}\right)^{2}}{r}\left\{-1+\frac{\omega e}{\hbar c^{2}} \frac{Q}{\alpha}\right\}
$$

if

$$
\varphi \cong \frac{Q}{r} \quad \text { and } \quad R \cong \frac{e^{-\alpha r}}{r} .
$$

Then

$$
\lim _{r \rightarrow \infty} \frac{d H}{d r}=0
$$

but it may be of either sign depending on the sign and magnitude of the second term. If the sign becomes positive after the representative point crosses the separatrix, it will settle into a closed orbit about either the left or right minimum, depending on whether it is caught in the left or right lobe. Then in configuration space $R$ will not approach a constant at infinity but will continue to oscillate.

We also note that the sign of $d H / d r$ may be switched outside the separatrix by slightly changing the magnitude of $\varphi$. If this happens when the representative point has come close in the approach to either lobe, it may happen that it finally terminates in the opposite lobe. In configuration space, this behavior will appear as a discontinuous change in the solution curve $R(r)$ caused by a small change in the parameter $\varphi(0)$. None of these remarks invalidate the exact nonperturbative procedure for determining the eigenvalues and eigensolutions of the coupled set of equations.

Since every solution is determined by the initial values $[R(0), \nu(0), \varphi(0)]$, the complete class of all solutions may be mapped onto a three-dimensional space with these coordinates. Each of these solutions must also be either a $p$ or an $s$ solution, as judged by the behavior of its $R$ component, unless it is an eigenfunction. It turns out that this threedimensional space may be divided into $p$ and $s$ cells, just as a three-dimensional crystal with two phases is divided into domains. The eigenvalues and corresponding eigenfunctions will then be located on the domain walls.

In order to look for numerical solutions for Eqs. (3.13), (3.17), (3.18), and (3.25) without the dilaton, we must recast these equations in dimensionless form 


$$
\begin{aligned}
\lambda^{\prime}= & \frac{\left(1-e^{\lambda}\right)}{x}-\frac{\delta}{2} x e^{\lambda}\left(\frac{\beta}{2} y^{2}-\frac{\gamma}{4} y^{4}+\frac{\gamma^{\prime}}{6} y^{6}\right) \\
& -\frac{\epsilon}{2} x\left(z^{\prime}\right)^{2} e^{-\nu}-\frac{\delta}{4} x\left[\left(y^{\prime}\right)^{2}+(\hat{\omega}+\alpha z)^{2} y^{2} e^{\lambda-\nu}\right],
\end{aligned}
$$

$$
\begin{aligned}
\nu^{\prime}= & -\frac{\left(1-e^{\lambda}\right)}{x}+\frac{\delta}{2} x e^{\lambda}\left(\frac{\beta}{2} y^{2}-\frac{\gamma}{4} y^{4}+\frac{\gamma^{\prime}}{6} y^{6}\right) \\
& +\frac{\epsilon}{2} x\left(z^{\prime}\right)^{2} e^{-\nu}-\frac{\delta}{4} x\left[\left(y^{\prime}\right)^{2}+(\hat{\omega}+\alpha z)^{2} y^{2} e^{\lambda-\nu}\right],
\end{aligned}
$$

$$
\begin{aligned}
y^{\prime \prime}= & {\left[\frac{\left(1-e^{\lambda}\right)}{x}-\frac{\delta}{2} x e^{\lambda}\left(\frac{\beta}{2} y^{2}-\frac{\gamma}{4} y^{4}+\frac{\gamma^{\prime}}{6} y^{6}\right)\right.} \\
& -\frac{\epsilon}{2} x\left(z^{\prime}\right)^{2} e^{-\nu}-\frac{2}{x}-\frac{\delta}{4} x\left[\left(y^{\prime}\right)^{2}+(\hat{\omega}\right. \\
& \left.\left.+\alpha z)^{2} y^{2} e^{\lambda-\nu}\right]\right] \\
& \times y^{\prime}-(\hat{\omega}+\alpha z)^{2} e^{\lambda-\nu} y+e^{\lambda}\left(\beta y-\gamma y^{3}+\gamma^{\prime} y^{5}\right),
\end{aligned}
$$

$$
\begin{aligned}
z^{\prime \prime}= & {\left[-\frac{\delta}{4} x\left[\left(y^{\prime}\right)^{2}+(\hat{\omega}+\alpha z)^{2} y^{2} e^{\lambda-\nu}\right]-\frac{2}{x}\right] z^{\prime} } \\
& -\frac{\alpha}{2} \frac{\delta}{\epsilon} e^{\lambda}(\hat{\omega}+\alpha z) y^{2},
\end{aligned}
$$

where prime stands for derivative with respect to $x$ as defined below. The different variables and parameters stand for

$$
\begin{aligned}
& \alpha=e^{2} / \hbar c, \\
& x=r / r_{0}, \quad \gamma^{\prime}=D r_{0}^{2} \bar{R}^{4}, \\
& \beta=B r_{0}^{2} \\
& y=R / \bar{R}, \quad \delta=K \bar{R}^{2}, \\
& \gamma=C r_{0}^{2} \bar{R}^{2} \\
& z=\varphi /\left(e / r_{0}\right), \quad \quad \epsilon=K\left(e / r_{0}\right)^{2}, \\
& \hat{\omega}=\left(\omega r_{0}\right) / c .
\end{aligned}
$$

In the previous equations $r_{0}$ is a fundamental length and $\bar{R}$ is a fundamental real field strength. There is a large set of solutions depending on the values of the parameters $\left(\beta, \gamma, \gamma^{\prime}, \delta, \epsilon, \hat{\omega}\right)$. With the boundary conditions (3.19)(3.21) and $\lambda(0)=0$ we can explore the differential equations for different initial values of the fields $R(0), \nu(0)$, and $\varphi(0)$. By the analysis of Sec. IV, the initial value $\varphi(0)$ is determined in terms of the initial value $R(0)$, via Eq. (4.12). Effectively we have a two-dimensional cut of the phase space of solutions depending on the initial values $R(0)$ and $\nu(0)$, which must be negative by Eq. (3.38).

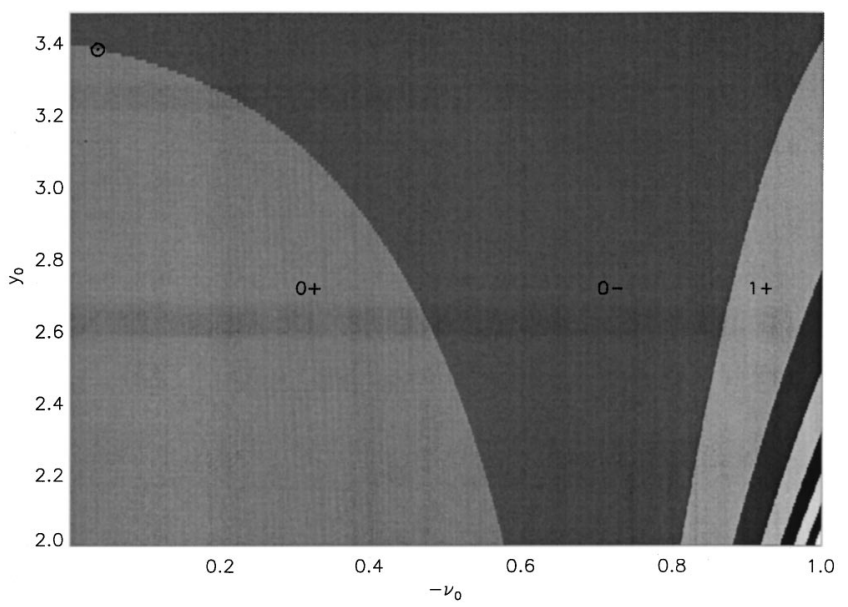

FIG. 4. Two-dimensional "phase diagram" in terms of the initial values $y(0)=R(0) / \bar{R}$ and $-\nu(0)$ for $z(0)=3.0$. The solutions are indicated by $n \pm$ where $n$ is the noding number and + and indicates whether the solution curve terminates in the $P$ or $S$ lobe. The eigensolution that is further explored in Fig. 5 and 6 is indicated by $\odot$. The values of the parameters in the numerical solutions are $\beta=0.001, \quad \gamma=0.0005, \quad \gamma^{\prime}=0.000038, \quad \hat{\omega}=0.002, \quad \delta=\epsilon$ $=-0.0001$ (since $K$ is negative).

In the following results the parameters are fixed at the following values: $\beta=0.001, \gamma=0.0005, \gamma^{\prime}=0.000038, \hat{\omega}$ $=0.002, \delta=\epsilon=-0.0001$ (since $K$ is negative). We vary the initial values $y(0)=R(0) / \bar{R}$ and $-\nu(0)$, with $z(0)$ $=\varphi(0) r_{0} / e=3$. As $y(0)$ increases and $\nu(0)$ decreases, we observe a transition from a non-noding solution that terminates at the attractor $S$ to one that terminates at $P$ (nonnoding). By Eq. (5.22) the eigensolution can be found in terms of the average of the two bounding solutions. If we continue to vary $y(0)$ and $\nu(0)$ we encounter the noding solutions that terminate at $S$ and $P$. In Fig. 4 we present this two-dimensional cut of the three-dimensional "phase space", in which the regions are labeled $n \pm$, where $n$ is the number of nodes, $(+)$ indicates termination at attractor $S$, and $(-)$ termination at attractor $P$. The boundary between $n+$ and $n-$ specifies the eigensolutions. Figure 5(a) presents an example of non-noding solutions $s$ and $p$ and their semisum which illustrates the approximate phase cancellation at large $r$. The initial values of the fields are $\nu(0)=-0.02, z(0)$ $=3.0$, and $y(0)=3.3922(s$ solution $) y(0)=3.3923(p$ solution).

The most important result of the numerical work is the observation that the $R(r)$ solutions divide into two classes when the four general relativistic differential equations are simultaneously integrated. This key feature of the special relativistic problem is exactly preserved. Now, however, associated with $R(p), R(s)$ and $R(q)$ are the families $[\lambda(p), \nu(p), \varphi(p)], \quad[\lambda(s), \nu(s), \varphi(s)]$, and $[\lambda(q), \nu(q)$, $\varphi(q)]$. Then in addition to Eq. (5.21), we have

$$
\lim _{p_{0}-s_{0} \rightarrow 0}\left[\frac{\lambda(p)+\lambda(s)}{2}\right]=\lambda(q)
$$



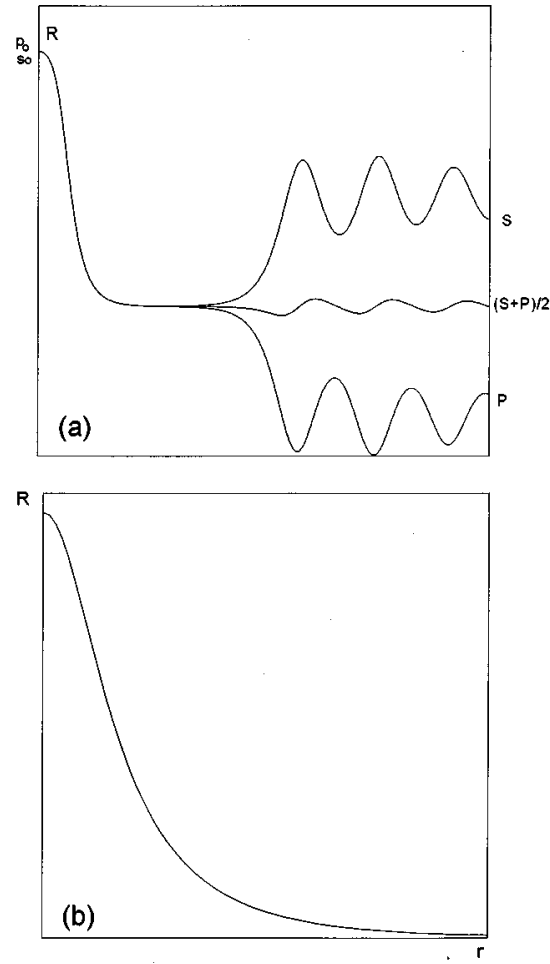

FIG. 5. (a) The asymptotic behavior of the solutions $s[y(0)$ $=3.3922]$ and $p[y(0)=3.3923]$, illustrating the approximate phase cancellation at large $r$. In this case $\nu(0)=-0.02$ and $z(0)=3.0$ and the values of the parameters are the same as for Fig. 4. (b) An eigensolution of $R(r)$ in configuration space determined by the numerical procedure described in the text.

with similar relations for $\nu$ and $\varphi$, so that in the limit $[R(q), \lambda(q), \nu(q), \varphi(q)]$ is a complete set of solutions corresponding to $q_{0}$. We also note that the $p$ and $s$ curves for $\lambda$, $\nu$, and $\varphi$ are nearly the same while the corresponding curves for $R$ are of course quite distinct at large $r$.

To perform the numerical analysis we follow the following approximate procedure. First carry the integration into the region where the gravitational potentials become asymptotic. Up to this point, say $\bar{r}$, which is somewhat arbitrary, approximate the four functions by their $s$ and $p$ average: $\frac{1}{2}[R(p)+R(s)], \frac{1}{2}[\lambda(p)+\lambda(s)], \ldots$. We shall choose as a criterion for determining $\bar{r}$ the condition

$$
\lambda(\bar{r})+\nu(\bar{r})=0 .
$$

Beyond this point approximate the eigensolution $R(q)$ by its asymptotic form (a spherical Bessel function). Then match the asymptotic form of $R(q)$ to $\frac{1}{2}[R(p)+R(s)]$ at $\bar{r}$ as follows:

$$
\begin{gathered}
\frac{a_{q} e^{-\alpha \bar{r}}}{\bar{r}}=\frac{1}{2}[R(p)+R(s)]_{\bar{r}}, \\
\left(\frac{a_{q} e^{-\alpha r}}{r}\right)_{\bar{r}}^{\prime}=\frac{1}{2}\left[R^{\prime}(p)+R^{\prime}(s)\right]_{\bar{r}} .
\end{gathered}
$$
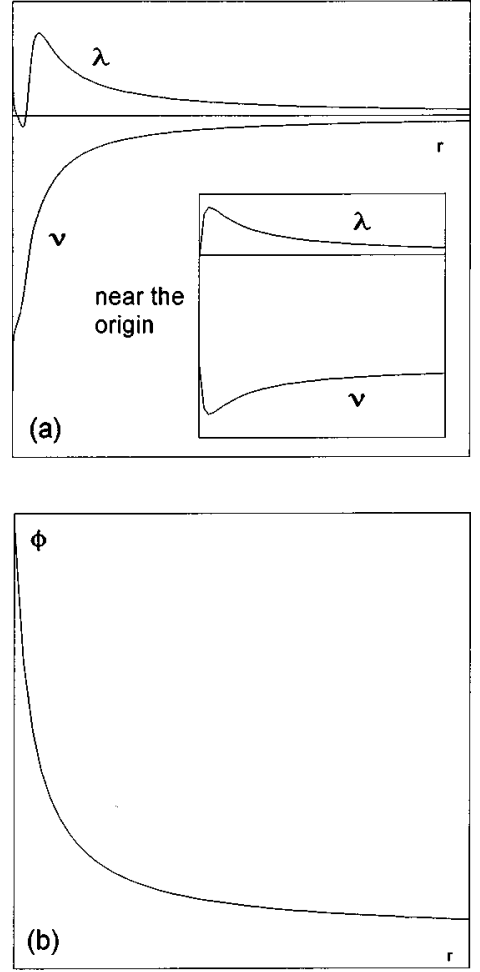

FIG. 6. (a) The gravitational eigensolutions $\lambda(r)$ and $\nu(r)$ obtained via the backward integration described in Sec. VI. (b) The electromagnetic eigensolution $\varphi(r)$.

To complete the integration beyond $\bar{r}$ feed the so obtained $R(q)$ into the complete set of differential equations while taking $\frac{1}{2}[\lambda(p)+\lambda(s)]_{\bar{r}} \ldots$ as initial values of the other functions. If the point defined by Eq. (6.12) is difficult to reach, one may instead choose $\bar{r}$ as the point at which the curves $s$ and $p$ begin to separate. An example of an eigensolution obtained by following the procedure described is presented in Fig. 5(b).

To find the functions $\varphi(r), \lambda(r)$, and $\nu(r)$, corresponding to the eigenfunction $R(r)$, we have integrated backwards by feeding $R(r)$ into the appropriate differential equations while imposing initial conditions determined by the asymptotic forms (4.29) and (4.30) and computed for a point $r_{\infty}$ and a mass $m$. The values of $r_{\infty}$ and $m$ must be adjusted to agree with the boundary conditions at $r=0$. The results of this backward integration are presented in Fig. 6 .

Additional checks may be obtained by integrating Eq. (3.13) and (3.25) as follows:

$$
\begin{aligned}
{[\nu(r)-\lambda(r)]_{0}^{\infty}=} & \int_{0}^{\infty} d r \frac{1}{r}\left(e^{\lambda}-1\right) \\
& +K \int_{0}^{\infty} d r r\left[V\left(R^{2}\right) e^{\lambda}+2 \Delta\right]=J_{1},
\end{aligned}
$$




$$
\begin{aligned}
{[\nu(r)+\lambda(r)]_{0}^{\infty}=} & -K \int_{0}^{\infty} d r r\left[\left(R^{\prime}\right)^{2}+\left(\frac{\omega}{c}+\frac{e}{\hbar c} \varphi\right)^{2}\right. \\
& \left.\times e^{\lambda-\nu} \frac{R^{2}}{c^{2}}\right]=J_{2}
\end{aligned}
$$

Now imposing the boundary conditions $\nu(\infty)=\lambda(\infty)=0$ we have

$$
\begin{aligned}
& \lambda(0)-\nu(0)=J_{1}, \\
& \lambda(0)+\nu(0)=-J_{2}<0 .
\end{aligned}
$$

To utilize these equations, let us note again that the solution is completely given by the initial values $[\lambda(0), \nu(0), \varphi(0), R(0)]$, the eigenvalues of the system. Of these we know that $\lambda(0)=0$ and that $\varphi(0)$ is approximately fixed by $R(0)$ according to Eq. (4.12), while $R(0)$ itself is determined as the solution of the non-linear problem that we have described. That leaves only $\nu(0)$ to be fixed and for this purpose Eqs. (6.17) and (6.18) may be reduced as follows:

Since $\lambda(0)=0$, we have

$$
\begin{aligned}
& -\nu(0)=J_{1}, \\
& -\nu(0)=J_{2} .
\end{aligned}
$$

If the trial function appearing in the $J_{1}$ and $J_{2}$ integrals are in fact exact eigenfunctions, we have $J_{1}=J_{2}=-\nu(0)$, but if they are not, then we may try

$$
-\nu(0)=\frac{1}{2}\left(J_{1}+J_{2}\right)
$$

as a next approximation to obtain new trial functions. One may continue to improve $\nu(0)$ by iterating Eq. (6.20).

The discrete spectrum of $R$ eigensolutions will induce discrete spectra on the other fields. As a consequence the mass of the soliton, which is identified by the coefficient of the $1 / r$ term in the asymptotic gravitational potential, is also limited to a discrete spectrum.

\section{DISCUSSION}

General relativistic solitons have also appeared in the literature on boson stars [5]. The purpose and methods of this paper are quite different, however, since they are more directly related to Ref. [1] and to the possible existence of nontopological solitons in fundamental completions of Einstein theory. The main result of the present work is the demonstration that the method of analysis employed in the earlier work may be extended to many coupled fields. While the field structures studied in this paper couple the gravitational, electromagnetic, nonlinear scalar, and dilaton fields, there are many other interesting possibilities that are tractable by the same method, such as gauge solitons and other structures generated by Kaluza-Klein-like theories.

Since there has been a particular emphasis in our investigation on the nonlinear scalar potential, the generality of this potential is of interest. Although a particular polynomial rep- resentation was chosen, it is clear that the analysis depends only on the distribution of minima and saddle points of the dissipative function along the field axis in phase space and therefore on the distribution of maxima and minima in the potential. One also notes that there are essentially only two kinds of potential, namely, the Higgs potential and inverted Higgs potential, or volcano, which are related by the interchange of minima with saddle points. If the potential is of the volcano type, there is a saddle point in the dissipative function flanked by two minima and if it is of the Higgs form, there is a central minimum bracketed by two saddle points. If this pattern of three extrema is centered at $r=0$, then the eigenstates of the former vanish at great distances. In the Higgs case there is a continuum of eigensolutions and in the case of the volcano potential there is a discrete set of eigensolutions, as we have discussed. If these patterns are not centered at $R=0$, however, then the corresponding eigenfunctions do not vanish at infinity. In this case one has the situation encountered in gauge theories where the limiting value at infinity is interpreted as a vacuum expectation value. If the potential is general with several minima, there should be both continuous and discrete spectra associated with it. For example, the potential may be Higgs-like at weak fields and volcanolike at strong fields. If the boundary conditions are interpreted in terms of vacuum expectation values, then the physical constants appearing in the field Lagrangian are encoded as vacuum expectation values. Since our remarks concerning the dilaton field may also be relevant to certain string models, let us consider two examples [6,7].

A dimensionally reduced superstring in four dimensions can be described in terms of $N=4$ supergravity: the action associated with the $S O(4)$ version of this theory is

$$
\begin{aligned}
I[\mathrm{SO}(4)]= & \int d^{4} x\left[-R+2 \partial^{\mu} \phi \partial_{\mu} \phi\right. \\
& \left.-\left(e^{-2 \phi} F_{\mu \nu} F^{\mu \nu}+e^{2 \phi} \widetilde{G}_{\mu \nu} \widetilde{G}^{\mu \nu}\right)\right]
\end{aligned}
$$

as expressed for example in Ref. [6]. Dropping the $G$ term one sees that the dilaton field $\phi$ appears here in the same way as in our equation (1.2). Therefore we may conclude that the same conclusion also holds here, namely, that nontopological solitons do not exist for this theory.

As a second example consider the five-brane soliton that is a solution of the field equations describing the low-energy heterotic string [7]. This soliton embodies a fourdimensional Yang-Mills instanton coupled to dilaton and axion fields according to superstring theory. The dilaton equation in this case is [7]

$$
\nabla \phi= \pm \frac{1}{120} \alpha^{\prime} \epsilon^{\mu \nu \rho \sigma} \operatorname{Tr} F_{\mu \nu} F_{\rho \sigma}
$$

This equation also resembles our equation (3.4) but with the following differences: the source term in Eq. (7.2) is pseudoscalar rather than scalar and moreover it lies in a gauge algebra; the instanton exists not in Minkowski but in Euclidean space, and the dilaton at spatial infinity does not 
vanish but approaches its vacuum expectation value. The field of this topological soliton is everywhere finite as required and its extension is determined by the size of the instanton. Its interpretation in conventional spacetime depends on how the five-branes wrap around topological nontrivial surfaces in the internal compactification manifold. Since this topological structure is therefore essentially differ-

[1] R. Finkelstein, R. Lelevier, and M. Ruderman, Phys. Rev. 83, 326 (1951).

[2] R. Friedberg, T. D. Lee, and A. Sirlin, Phys. Rev. D 13, 2739 (1976).

[3] R. Friedberg, T. D. Lee, and Y. Pang, Phys. Rev. D 35, 3640 (1987); 35, 3658 (1987). ent from the nontopological four-dimensional solution studied here, our remarks about the dilaton field do not apply to this example although they do to the preceding case. On the other hand, the procedure described here may be useful for investigating non-Abelian as well as Abelian theories, and therefore for describing topological as well as nontopological solitons.
[4] A. C. Cadavid and R. J. Finkelstein, Phys. Rev. D 57, 7318 (1998).

[5] P. Jetzer, Phys. Rep. 220, 163 (1992).

[6] R. Kallosh, A. Linde, T. Ortin, A. Peet, and A. Van Proyen, Phys. Rev. D 46, 5278 (1992).

[7] A. Strominger, Nucl. Phys. B343, 167 (1990). 